\title{
A bound iron porphyrin is redox active in hybrid bacterial reaction centers modified to possess a four-helix bundle domain
}

\author{
J. P. Allen ${ }^{1} \oplus$ - K. D. Chamberlain ${ }^{1} \cdot$ T. L. Olson ${ }^{1}$ - J. C. Williams ${ }^{1}$
}

Received: 29 September 2021 / Accepted: 16 November 2021 / Published online: 30 November 2021

(c) The Author(s), under exclusive licence to European Photochemistry Association, European Society for Photobiology 2021

\begin{abstract}
In this paper we report the design of hybrid reaction centers with a novel redox-active cofactor. Reaction centers perform the primary photochemistry of photosynthesis, namely the light-induced transfer of an electron from the bacteriochlorophyll dimer to a series of electron acceptors. Hybrid complexes were created by the fusion of an artificial four-helix bundle to the M-subunit of the reaction center. Despite the large modification, optical spectra show that the purified hybrid reaction centers assemble as active complexes that retain the characteristic cofactor absorption peaks and are capable of light-induced charge separation. The four-helix bundle could bind iron-protoporphyrin in either a reduced and oxidized state. After binding iron-protoporphyrin to the hybrid reaction centers, light excitation results in a new derivative signal with a maximum at $402 \mathrm{~nm}$ and minimum at $429 \mathrm{~nm}$. This signal increases in amplitude with longer light durations and persists in the dark. No signal is observed when iron-protoporphyrin is added to reaction centers without the four-helix bundle domain or when a redox-inactive zinc-protoporphyrin is bound. The results are consistent with the signal arising from a new redox reaction, electron transfer from the iron-protoporphyrin to the oxidized bacteriochlorophyll dimer. These outcomes demonstrate the feasibility of binding porphyrins to the hybrid reaction centers to gain new light-driven functions.
\end{abstract}

\section{Graphical abstract}

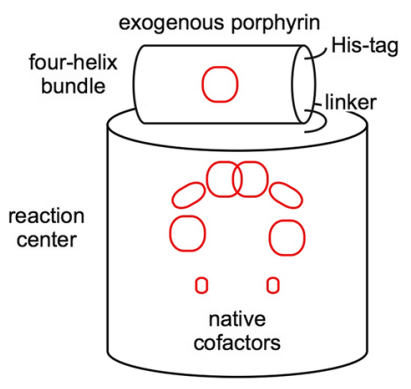

Keywords Photosynthesis $\cdot$ Electron transfer $\cdot$ Protein design $\cdot$ Hemes $\cdot$ Synthetic biology

Themed issue on artificial, semiartificial, and hybrid photosynthesis.

\footnotetext{
J. P. Allen

jallen@asu.edu

1 School of Molecular Sciences, Arizona State University, Tempe, AZ 85287, USA
}

\section{Introduction}

The incorporation of synthetic molecules into proteins results in new directions to probe the cellular functions of natural proteins and also enables the design of functional artificial proteins [1-6]. The design of artificial proteins has made tremendous progress in many areas, notably in the ability to achieve stable configurations having well-defined secondary structures with the resulting proteins mimicking existing natural proteins as well as adopting novel folds not 
found in nature $[7,8]$. The inclusion of synthetic molecules that facilitate specific functions in hybrid proteins with artificial and natural domains remains a scientific challenge.

Natural proteins that perform catalysis or electron transfer reactions often possess metal and tetrapyrrole cofactors as found for many key oxidation/reduction reactions in nature, such as biosynthetic processes, nitrogen fixation, and photosynthesis. The design of artificial proteins with these types of cofactors requires manipulation to create metal ligands and suitable binding pockets [9-12]. One design that has proven to be a stable and robust scaffold for the binding of cofactors is the four-helix bundle, and several groups have established experimental conditions to tightly bind a range of metal clusters as well as hemes and other tetrapyrroles [13-17]. For example, we have examined the ability of fourhelix bundles to bind Mn centers [18-20]. Tetrapyrroles can be bound tightly to four-helix bundles with dissociation constants less than $1 \mu \mathrm{M}$ with their positioning determined by the incorporation of coordinating His residues [13]. We are using these studies to provide a foundation for the utilization of four-helix bundles capable of binding tetrapyrroles to investigate electron transfer reactions.

Previously, we have examined the ability of four-helix bundles with bound metal cofactors to transfer electrons to bacterial reaction centers. Reaction centers are membranebound pigment-protein complexes that perform the primary photochemistry in bacterial photosynthesis [21, 22]. In the reaction center from Rhodobacter sphaeroides, light absorption by a bacteriochlorophyll dimer, $\mathrm{P}$, initiates the transfer of an electron from $\mathrm{P}$ through intermediate electron acceptors to the primary quinone, $\mathrm{Q}_{\mathrm{A}}$, and then the secondary quinone, $\mathrm{Q}_{\mathrm{B}}$. To have the chemical capability to drive metal oxidation, $\mathrm{P}$ was modified in the highly-oxidizing $\mathrm{T} 1 \mathrm{reac}-$ tion centers by the replacement of three amino acid residues, Leu M160, Leu L131 and Phe M197 with His, resulting in the addition of three new hydrogen bonds to the conjugated system of $\mathrm{P}$ [23]. The $\mathrm{T} 1$ reaction centers were able to oxidize, as second-order processes, dinuclear Mn-clusters bound to four-helix bundle proteins $[18,20]$. The bundle was modeled as transiently docking to the periplasmic surface of the reaction center at a location similar to that of the natural secondary donor, cytochrome $c_{2}$, driven by a range of electrostatic and hydrophobic interactions between the two proteins.

In this paper, we demonstrate that a redox-active porphyrin cofactor can be incorporated into a hybrid bacterial reaction center modified to possess a four-helix bundle domain (Fig. 1). The basis for the design of our four-helix bundle is the due ferri (DF) protein family of artificial proteins that form four-helix bundles with central metal cofactors, specifically in this case from a single polypeptide chain [24-26]. Linking the bundle protein genetically to the C-terminus of the $\mathrm{M}$ subunit of the reaction center should position the

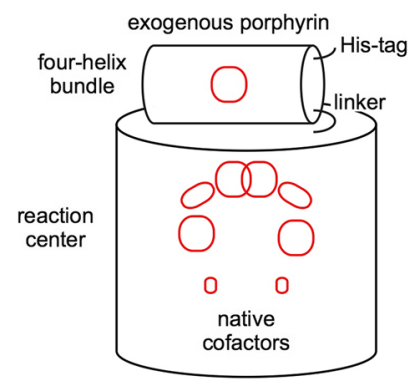

Fig. 1 Features of the hybrid protein MPB2. An artificial four-helix bundle is linked to the reaction center protein. Exogenous porphyrins can be incorporated into the added domain, which includes a C-terminal His-tag to ensure the purification of the intact fusion protein. The reaction center cofactors form an electron transfer chain that is initiated by light, resulting in an oxidized bacteriochlorophyll dimer, whose redox properties are adjusted with modifications to nearby amino acid residues

four-helix domain on the periplasmic surface, close to the bacteriochlorophyll dimer. Because the hybrid protein, termed MPB2, is isolated without a bound cofactor in the four-helix bundle domain, an exogenous cofactor, such as a porphyrin, can be added. The initiation of electron transfer by light produces an oxidized bacteriochlorophyll dimer that could interact with a redox-active porphyrin. Since porphyrins have rich optical spectra, their electronic states were monitored using optical spectroscopy to identify new electron transfer reactions in the hybrid reaction centers.

\section{Methods}

\subsection{Preparation of four-helix bundle and fusion proteins}

To construct the genes for the PB1 single-chain four-helix bundle and the MPB2 fusion of the reaction center with the four-helix bundle, restriction fragments encoding the fourhelix bundle and the C-terminal extension of the $\mathrm{M}$ subunit were synthesized and inserted into previously described plasmids (Fig. S1). The PB1 portion was inserted into the expression vector pET30a + and expressed in E. coli BL21(DE3) (Novagen). The MPB2 fragment was incorporated into the $\mathrm{M}$ subunit gene, and then into a broad-hostrange plasmid with the rest of the puf operon, and expressed in a strain of $R$. sphaeroides in which the wild-type genes had been deleted [27].

The MPB2 fusion protein was isolated using Ni-affinity chromatography $[28,29]$. In some cases, the MPB2 reaction centers were further purified by protease cleavage and anion exchange chromatography. The wild-type reaction centers and previously described $\mathrm{T} 1$ reaction centers were isolated using ion-exchange chromatography $[23,30]$. The 
$\mathrm{PB} 1$ protein was isolated using Ni-affinity chromatography followed by protease cleavage as previously described [19, 20]. The concentration of the final PB1 protein consisting of 115 amino acid residues was determined using a calculated extinction coefficient of $8480 \mathrm{M}^{-1} \mathrm{~cm}^{-1}$ at $280 \mathrm{~nm}$. Sodium dodecyl sulfate-polyacrylamide gel electrophoresis (SDS-PAGE) with a 4-15\% gradient Mini-PROTEAN TGX precast gel (BioRad, USA) was used to assess the purity and sizes of the isolated proteins. Amicon $3000 \mathrm{Da}$ and 45,000 Da molecular weight cutoff filters were used for washing and buffer exchange of the PB1 and MPB2 proteins, respectively. Stocks of iron protoporphyrin IX (porcine hemin, Sigma-Aldrich), and zinc protoporphyrin IX (Alfa Aesar) were made in DMSO, and then added to solutions of the PB1 or MPB2 proteins and incubated at room temperature for $30 \mathrm{~min}$ in an $\mathrm{N}_{2}$ atmosphere, following the general guidelines in published protocols [13-15, 17, 31].

\subsection{Spectroscopic measurements}

Steady-state and transient absorption measurements were performed using either a Cary 50 BIO or a Cary 6000i spectrophotometer (Agilent Technologies, Inc., USA). Lightinduced spectral changes were measured with the reaction centers illuminated using sub-saturating light from a 1000-W tungsten bulb with an $860 \mathrm{~nm}$ interference filter as previously described [30]. Samples were typically illuminated for a total of $90 \mathrm{~s}$ as the wavelength was swept from 1000 to $350 \mathrm{~nm}$. Prior to the measurements, the buffer was changed to $15 \mathrm{mM}$ 2-(cyclohexylamino)ethanesulfonic acid (CHES) pH 9.4 for the PB1 samples and to 15 mM CHES pH 9.4, $0.8 \%$ octyl- $\beta$-D-glucopyranoside for the MPB2 samples. Reaction centers were measured at $2 \mu \mathrm{M}$ with the porphyrins at $2-10 \mu \mathrm{M}$. The PB1 protein was used at $10 \mu \mathrm{M}$ with $10-20 \mu \mathrm{M}$ porphyrin. For spectra of proteins with reduced iron-protoporphyrin, $100 \mu \mathrm{M}$ sodium dithionite was added. Some measurements were made in the presence of 100-300 $\mu \mathrm{M}$ terbutryn.

Circular dichroism spectroscopy was performed on a $\mathrm{J}-815$ spectropolarimeter equipped with a thermostatic cell holder (JASCO, Japan). The spectra were measured from 260 to $190 \mathrm{~nm}$ using a $0.1 \mathrm{~cm}$ path length quartz cuvette at $4{ }^{\circ} \mathrm{C}$. The scan rate was $50 \mathrm{~nm} / \mathrm{min}$ with $0.5 \mathrm{~nm}$ increments. The PB1 protein concentration was $7.5 \mu \mathrm{M}$ in $7.5 \mathrm{mM}$ 4-(2-hydroxyethyl) piperazine-1-ethanesulfonic acid (HEPES) $\mathrm{pH}$ 7.5. For reaction centers, the protein was in $5 \mathrm{mM}$ Tris $\mathrm{pH} 8$ and $0.05 \%$ LDAO and the concentration was adjusted in the $0.07-0.4 \mu \mathrm{M}$ range to achieve spectra with amplitudes of 10-15 millidegrees at $190 \mathrm{~nm}$. The spectra were analyzed using the programs SELCON 3, CDSSTR, CONTINLL, and K2D3 [32-35]. Only the data in the 190-240 $\mathrm{nm}$ range were used for analysis.

\section{Results}

\subsection{Design and construction of reaction center fusion}

In the hybrid protein, we have fused a four-helix bundle with a porphyrin binding site to the reaction center. For comparison, we have also isolated separately the four-helix bundle and the reaction center forming the base of the fusion protein. The hybrid protein contains a modified $\mathrm{M}$ subunit sequence that is followed by a short linker and a four-helix bundle (Fig. 2). The Gly linker has the sequence GGNGGN [36] and continues from the C-terminal residue of the wildtype $\mathrm{M}$ subunit, shortly after the transmembrane helix $\mathrm{E}$ of the reaction center. Modeling and energy minimization show that the linker can form a loop resulting in the placement of bundle domain on the periplasmic surface with two helices of the four-helix bundle near the bacteriochlorophyll dimer. The single-chain four-helix bundle sequence was based on the DFsc protein [24, 37, 38]. The His ligands shown to bind metal and porphyrin cofactors are found in two of the helices. A C-terminal cleavable His-tag is used for isolation and to ensure that the purified reaction centers contain the extension.

The reaction center hybrid designated MPB2 has the mutations L131 Leu to His, M160 Leu to His, and M197 Phe to His found in the highly-oxidizing T1 reaction centers as well as a cleavable His-tag present at the end of the four-helix bundle sequence of the extended $\mathrm{C}$-terminus. The four-helix bundle protein designated PB 1 contains the same sequence as in the M subunit of the MPB2 reaction centers starting from immediately after the linker but lacking the C-terminal cleavage site and His-tag.

\subsection{Binding of iron-protoporphyrin to four-helix bundle in reaction center fusion}

After expression in $R$. sphaeroides, solubilization from the membrane, and purification by Ni-affinity chromatography, the average yield of the fusion protein MPB2 reaction centers was found to be approximately $30 \%$ of that

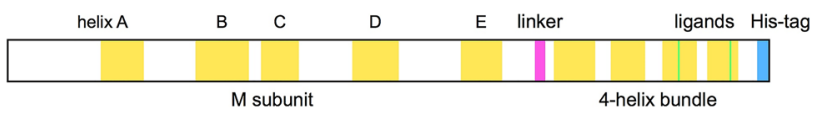

...GFNATMEGIHRWAIWMAVLVTLTGGIGILLSGTVVDNWYVWGQNHGMAPLNGGNGGNGDELRELLKAEQQAIKIYKEVLKKAKEGDEQELARLIQEIVKAEKQAVKVYKEAAEKARNPEKRQVIDKILEDEEKHIEWLKAASKQGNAEQFASLVQQILQDEQRHVEEIEKKNENLYFQGHHHHHHH

Fig. 2 Diagram of the M subunit fused to the artificial four-helix bundle and C-terminal sequence of the reaction center M-subunit fusion. The locations of the linker (pink), cofactor ligands (green) and Histag (blue) are indicated relative to the helices (yellow) 
obtained for preparations of wild-type reaction centers. The purified hybrid reaction centers had typical absorption spectra with bacteriopheophytin and bacteriochlorophyll absorption bands, including an absorption band centered at $865 \mathrm{~nm}$ that is associated with $\mathrm{P}$. These absorption bands match the wavelength positions and amplitudes characteristic of the $\mathrm{T} 1$ reaction centers, indicating that they retained a normal arrangement of the cofactors (Fig. 3a). The sizes of the three protein subunits of the reaction center, $\mathrm{L}, \mathrm{M}$, and $\mathrm{H}$, were evaluated using SDS-PAGE. The $\mathrm{L}$ and $\mathrm{H}$ subunits had comparable sizes but the band associated with the M subunit of the MPB2 reaction centers was shifted compared to wild type, corresponding to the additional $\sim 15 \mathrm{kDa}$ domain (Fig. S2a).

Light excitation of $\mathrm{T} 1$ reaction centers results in the formation of the charge-separated state that has characteristic spectral differences compared to the ground state, including loss of absorption of the $865 \mathrm{~nm}$ band. These spectral changes are also present when the MBP2 reaction centers were illuminated, demonstrating that the hybrid reaction centers are active (Fig. 4a). The yield of electron transfer as reflected in the extent of the absorption change of the $\mathrm{P}$ band was comparable to other reaction centers with the mutations that result in high $\mathrm{P} / \mathrm{P}^{+}$midpoint potentials [39].
The secondary structures of the purified four-helix bundles and fusion reaction centers were characterized using circular dichroism (Fig. S2b). The four-helix bundle had a spectrum similar to previous measurements and yielded a large $\alpha$-helix component $[19,37]$. The reaction centers without the fusion bundle showed spectra with a $\sim 55 \% \alpha$-helix component as previously reported [40, 41]. The MPB2 reaction centers had a slightly higher $\alpha$-helix content than the non-hybrid reaction centers, consistent with the additional sequence region folding as a four-helix bundle.

Binding of iron-protoporphyrin was tested with the PB1 four-helix bundle. The PB1 protein allows measurement of the porphyrin optical spectra alone, as the MPB2 fusion protein contains overlapping absorption bands from the bacteriopheophytin and bacteriochlorophyll cofactors (Fig. 3). After incubation of iron-protoporphyrin with the PB1 protein, the sample (PB1-FeP) yielded distinctive spectral features that are typical of the oxidized and reduced states of tetrapyrroles bound to four-helix bundles and other proteins $[13,42]$. The spectra of the PB1-FeP protein were similar before and after washing the sample, while the spectrum of samples of the iron-protoporphyrin in the buffer without the PB1 protein lost all spectral features after washing, showing that the iron-protoporphyrin binds tightly to the $\mathrm{PB} 1$ protein. The spectrum of the $\mathrm{PB} 1-\mathrm{FeP}$ protein has a
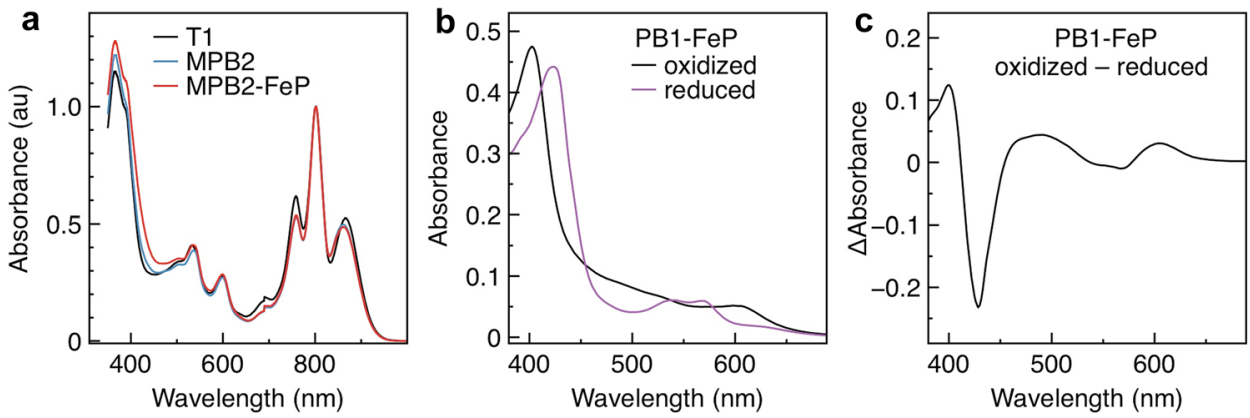

Fig. 3 Absorption spectra of reaction centers and four-helix bundle with iron-protoporphyrin. a Normalized spectra of isolated T1 and MPB2 reaction centers, and MPB2 reaction centers with iron-protoporphyrin bound (MPB2-FeP). b Spectra of the four-helix bundle

PB1 with iron-protoporphyrin bound (PB1-FeP) and after addition of sodium dithionite. c Difference spectra of PB1-FeP (oxidized) and $\mathrm{PB} 1-\mathrm{FeP}$ with dithionite (reduced)
Fig. 4 Light-minus-dark difference spectra for MPB2 and MPB2-FeP reaction centers in the presence of terbutryn. Shown are the spectra a during a $90 \mathrm{~s}$ total illumination period, b after $5 \mathrm{~min}$ in the dark, and c upon illumination times of $90 \mathrm{~s}, 180 \mathrm{~s}, 270 \mathrm{~s}$, and $360 \mathrm{~s}$ of MPB2-FeP reaction centers, with each spectrum taken sequentially
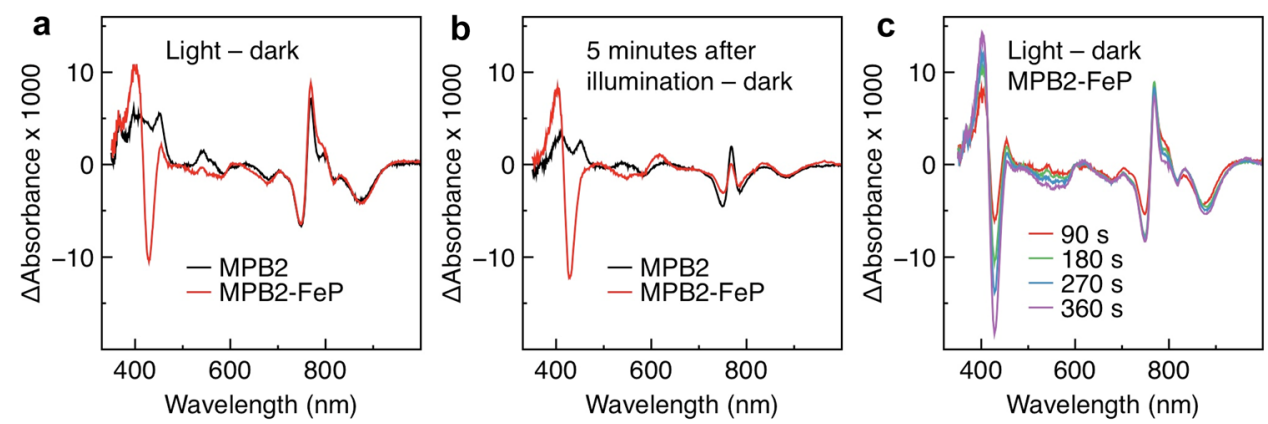
Soret band at $403 \mathrm{~nm}$ and a smaller band at $605 \mathrm{~nm}$ consistent with a bound iron-protoporphyrin in the oxidized state. The fully reduced state of the porphyrin was generated by the addition of dithionite, and in this case the Soret band shifted to $424 \mathrm{~nm}$, with resolved bands at 535 and $570 \mathrm{~nm}$. These spectra were subtracted to predict the oxidized-minusreduced spectrum, producing a derivative shape with a peak at $390 \mathrm{~nm}$ and a minimum at $429 \mathrm{~nm}$, and providing a clear spectral marker for a redox change of a tetrapyrrole bound to the MPB2 hybrid protein.

For the binding of iron-protoporphyrin to the MPB2 reaction centers (MPB2-FeP), the iron-protoporphyrin absorption bands were not distinctive (Fig. 3a). The spectrum of the MBP2-FeP reaction centers did not change after washing to remove unbound porphyrin. Subtraction of the spectrum of the MPB2 reaction centers with iron-protoporphyrin from MPB2 reaction centers without iron-protoporphyrin yielded the spectral contributions of iron-protoporphyrin in the oxidized and reduced states, showing similar peaks to those for PB1-FeP protein (Fig. S3).

\subsection{Light-induced optical changes}

In the presence of light, wild-type reaction centers perform charge separation by electron transfer from the excited state of $\mathrm{P}$ to the primary and secondary quinones. Accompanying the formation of the charge-separated states are changes in the absorption spectra compared to the ground state. These spectral changes are revealed by light-minus-dark optical spectra whose major features are an absorption decrease of the $\mathrm{P}$ band near $865 \mathrm{~nm}$, associated with the formation of $\mathrm{P}^{+}$, and electrochromic shifts of the three peaks in the near-IR region due to the presence of the reduced quinone $\left(\mathrm{Q}_{\mathrm{A}}^{-}\right)$, along with smaller changes in the visible region, as was observed for the MPB2 reaction centers in the presence of terbutryn (Fig. 4a).

When iron-protoporphyrin was bound to the MPB2 reaction centers (MPB2-FeP), a new signal in the visible region was observed, with a derivative shape having a minimum at $429 \mathrm{~nm}$ and a maximum at $402 \mathrm{~nm}$, in addition to the spectral features associated with the formation of the charge-separated state (Fig. 4a). The shape and position of this signal were similar to the calculated oxidized-reduced spectrum of the PB1-FeP protein (Fig. 3c). The derivative feature overlaps a broad rise at $450 \mathrm{~nm}$ that was observed in the absence of the porphyrin. Small increases in the $\mathrm{Q}_{\mathrm{A}}{ }^{-}$electrochromic shifts were also observed in the MPB2FeP reaction centers compared to the MPB2 reaction centers.

After the end of the illumination, charge recombination back to the ground state results in a reduction in the amplitudes of the spectral features associated with the chargeseparated state (Fig. 4a). However, the derivative feature associated with iron-protoporphyrin persisted with only minor changes in amplitude after five minutes in the dark (Fig. 4b). Signals associated with the charge-separated state that remain in the MPB2 reaction centers after five minutes in the dark, both with and without iron-protoporphyrin, are indicative of a fraction of the reaction centers with slow charge recombination after the continuous illumination conditions. Additionally, in some cases small broad losses of absorption centered at approximately $780 \mathrm{~nm}$ and $870 \mathrm{~nm}$ were observed after illumination, indicating an apparent light-induced degradation of the tetrapyrrole pigments (Fig. S4).

To test the stability of the $402 / 429 \mathrm{~nm}$ signal, the spectrum of the MPB2-FeP reaction centers was measured under prolonged illumination. Illumination of the MPB2-FeP reaction centers for $90,180,270$, and 360 s resulted in successive increases in the amplitude of the derivative signal at $402 / 429 \mathrm{~nm}$, while the amplitudes of the other absorption bands did not show large changes (Fig. 4c). Thus, the yield of the derivative signal at $402 / 429 \mathrm{~nm}$ could be accumulated with additional light exposure.

The fused four-helix bundle was required for the observed light-induced changes in the MPB2-FeP reaction centers, as demonstrated by comparison with $\mathrm{T} 1$ reaction centers. The $\mathrm{T} 1$ reaction centers have the same base reaction center as the MPB2 reaction centers but the $\mathrm{T} 1$ reaction centers do not contain the four-helix bundle domain, and hence no binding site for a porphyrin. In the light, the $\mathrm{T} 1$ reaction centers have a spectrum corresponding to the presence of $\mathrm{P}^{+} \mathrm{Q}^{-}$(Fig. 5a). This spectrum matches the features of wild type, with smaller
Fig. 5 Light-minus-dark difference spectra for T1 and MPB2 reaction centers. a $\mathrm{T} 1$ reaction centers with and without iron-protoporphyrin added as an exogenous molecule. b MPB2 reaction centers with and without bound zinc-protoporphyrin. c MPB2 reaction centers with and without bound iron-protoporphyrin
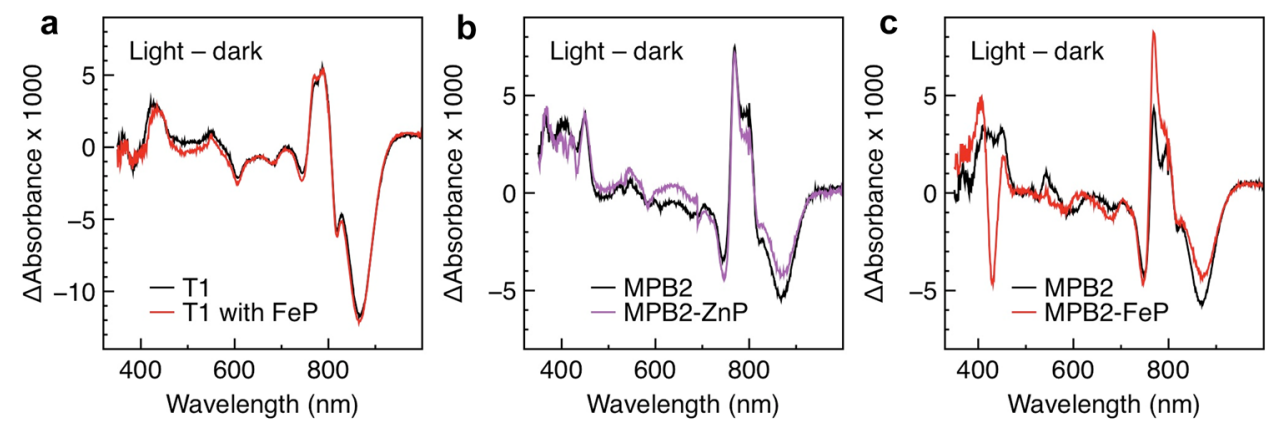
amplitudes due to the higher redox potential for $\mathrm{P}$ in $\mathrm{T} 1$ reaction centers compared to wild type. The addition of iron-protoporphyrin to the $\mathrm{T} 1$ reaction centers resulted in only minor changes in the light-minus-dark spectrum. For the T1 reaction centers both with and without iron-protoporphyrin, the spectra recovered after $10 \mathrm{~min}$ in the dark. Thus, the addition of iron-protoporphyrin to highly oxidizing reaction centers is insufficient to observe the derivative signal at $402 / 429 \mathrm{~nm}$.

In contrast to the results with iron-protoporphyrin, lightminus-dark measurements performed on MPB2 reaction centers with zinc-protoporphyrin resulted in only very minor alterations of the spectra (Fig. 5b). The presence of this redox-inactive porphyrin being insufficient to observe a derivative signal indicates that a change in redox state is required, supporting the interpretation that the derivative signal at $402 / 429 \mathrm{~nm}$ arises due to redox changes of iron-protoporphyrin.

In the MPB2-FeP reaction centers, under continuous illumination, several electron-transfer turnovers can take place because of the ability of reaction centers to accumulate two electrons on the secondary quinone and one electron on the primary quinone, so that $\mathrm{P}^{+}$can be regenerated after reduction of $\mathrm{P}^{+}$by the bound iron-protoporphyrin or by $\mathrm{P}^{+} \mathrm{Q}^{-}$charge recombination. Addition of terbutryn blocks electron transfer to the secondary quinone, and hence only one electron can be transferred to the primary quinone. In the MPB2 reaction centers without terbutryn, the predominate spectral change due to the addition of iron-protoporphyrin was the presence of the derivative signal at $402 / 429 \mathrm{~nm}$ (Fig. 5c), similar to the signal observed in the presence of terbutryn (Fig. 4a).

In light-minus-dark spectra, the amplitude of the derivative signal at 402/429 $\mathrm{nm}$ increased with repeated light exposure (Fig. 4c). The kinetics of this phenomenon was observed by monitoring the absorption at $429 \mathrm{~nm}$ (Fig. 6). For both MPB2 and MPB2-FeP reaction centers, immediately after the start of illumination a small increase in absorbance was observed. However, after this initial increase the two samples had contrasting behavior. For the MPB2 reaction centers without iron-protoporphyrin, the absorbance increased, while the absorbance for MPB2-FeP reaction centers decreased with a time constant of approximately $1 \mathrm{~min}$. At the end of illumination, both samples showed a small decrease, which was followed by a decrease for the MPB2 reaction centers while the absorbance of the MPB2FeP reaction centers changed very slowly.

\section{Discussion}

In nature, reaction centers absorb light to reduce quinones through a series of proton-coupled electron transfer processes that have proven to be amenable to manipulation.

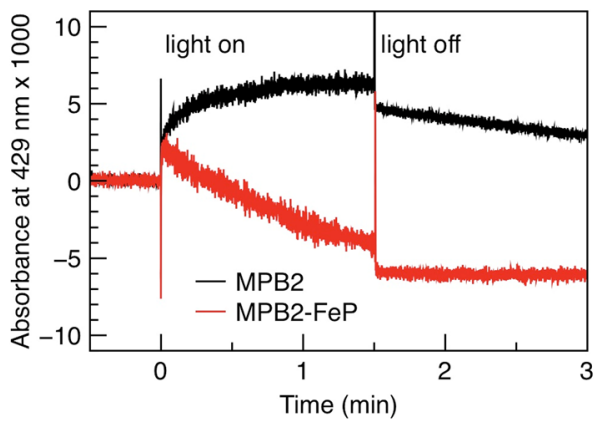

Fig. 6 Kinetics of the signal associated with oxidation of the iron porphyrin measured at $429 \mathrm{~nm}$ in MPB2-FeP reaction centers. In the absence of iron-protoporphyrin, a small rise was observed in the light, but when iron-protoporphyrin was bound to the reaction centers, a steady decrease at $429 \mathrm{~nm}$ in the light was observed

Previous studies have used mutagenesis to examine the role of the protein environment in establishing these electron transfer properties [39, 43]. In some cases, alterations of the amino acid residues coordinating cofactors have resulted in the binding of different cofactors, for examining the alteration of a His that coordinates P results in the incorporation of a bacteriopheophytin-bacteriochlorophyll heterodimer [44-46]. Our laboratory is making use of an alternative strategy of altering the protein to provide binding sites for new cofactors that could potentially introduce new electron transfer reactions. In earlier studies, Mn-binding sites were incorporated near a highly-oxidizing P resulting in reaction centers capable of binding redox-active Mn-cofactors that could rapidly reduce $\mathrm{P}^{+}[29,30]$. While useful for the examination of new metal cofactors, those studies were limited to configurations allowed by the alterations of existing amino acid residues.

In this study, we have taken the approach of introducing a large new protein domain, consisting of over 100 amino acid residues that form a four-helix bundle with a linker that directly fuses the bundle to the $\mathrm{C}$-terminus of the $\mathrm{M}$-subunit of the reaction center (Fig. 2). The resulting MPB2 reaction centers were isolated using a His-tag located at the C-terminus of the bundle ensuring that the purified protein contained the entire modified M-subunit, precluding the possibility of post-translational processing in the periplasm. The absorption and light-minus-dark optical spectra indicate that the purified reaction centers are functional, with the same configuration of cofactors and electron transfer activity as typically found in wild-type and the highly-oxidizing T1 mutant reaction centers (Figs. 3 and 4). The availability of the purified MPB2 reaction centers with a four-helix bundle domain on the donor side provided the capacity to introduce novel cofactors at designed binding sites.

Both the PB1 four-helix bundle protein and the MPB2 reaction centers containing the four-helix bundle fusion 
show optical spectra that correspond to iron-protoporphyrin even after washing off the protein, indicating that the iron-protoporphyrin is bound to the four-helix bundle. Illumination of the MPB2-FeP reaction centers resulted in the appearance of a derivative signal at $402 / 429 \mathrm{~nm}$, which persisted after the end of illumination (Fig. 4). The derivative signal did not appear in either reaction centers that lacked the four-helix bundle or in MPB2 reaction centers with zincprotoporphyrin, confirming that the signal results from a redox active iron-protoporphyrin bound to the added fourhelix bundle domain (Fig. 5). The 402/429 nm derivative signal corresponds to the oxidation of iron-protoporphyrin, making it likely that the MPB2 reaction centers bind a mixture of oxidized and reduced iron-protoporphyrin. This feature can be interpreted as an electron being transferred from iron-protoporphyrin to reduce $\mathrm{P}^{+}$, producing a stable, oxidized iron-protoporphyrin. Alternatively, the signal may result from oxidation of a different pigment in the reaction center in a reaction that requires the presence of the bound iron-protoporphyrin, for example one that results in the creation of an oxygen radical. While elucidation of the precise mechanism would require additional experiments, the spectroscopic results provide clear evidence that ironprotoporphyrin binds to the four-helix domain of the MPB2 reaction centers as a redox-active cofactor that participates in the light-induced electron transfer processes.

Although the iron-protoporphyrin bound to the fourhelix bundle appears to act as a secondary donor to the reaction center, it is much less efficient than the native secondary donor in $R$. sphaeroides, cytochrome $c_{2}$, which is a mobile electron carrier that transiently docks onto the reaction center. Based upon the three-dimensional structure of cytochrome $c_{2}$ bound to the reaction center, the heme cofactor reduces $\mathrm{P}^{+}$from a distance of $8.4 \AA$ between closest atoms [47]. The (cytochrome $\left.c_{2}\right)^{2+} /\left(\text { cytochrome } c_{2}\right)^{3+}$ midpoint potential is about $350 \mathrm{mV}$, while the $\mathrm{P} / \mathrm{P}^{+}$midpoint potential of wild-type reaction centers is about $500 \mathrm{mV}$, providing a sufficient energy difference for rapid electron transfer with a rate of electron transfer of $10^{6} \mathrm{~s}^{-1}$ from the bound cytochrome $c_{2}$ to reduce $\mathrm{P}^{+}$[23]. For iron-protoporphyrin bound to four-helix bundles, the midpoint potentials for conversion between ferrous porphyrin and ferric porphyrin range from -90 to $-290 \mathrm{mV}[13,14,42]$. Assuming a value in this range for the midpoint potential of iron-protoporphyrin bound to MPB2, the energy difference should be more than sufficient for electron transfer from iron-protoporphyrin to $\mathrm{P}^{+}$. However, based on the small amplitude of the optical changes, the yield of the formation of the oxidized ironprotoporphyrin was relatively low.

Oxidation of the iron-protoporphyrin occurs after the production of the $\mathrm{P}^{+} \mathrm{Q}^{-}$charge-separated state. The experiments were performed using continuous illumination at $860 \mathrm{~nm}$ that generates the excited state of $\mathrm{P}$, which is followed by the formation of the $\mathrm{P}^{+} \mathrm{Q}^{-}$charge-separated state. Since the porphyrins absorb only in the visible region, they are not excited by the $860 \mathrm{~nm}$ light. The rate of iron-protoporphyrin oxidation competes with charge recombination from the quinones, which has a time of approximately $40 \mathrm{~ms}$ in the reaction centers with a high $\mathrm{P} / \mathrm{P}^{+}$midpoint potential [23]. In addition, continuous illumination of reaction centers results in the production of recovery kinetics that become slower and multiphasic compared a single laser flash [48]. Complex $\mathrm{P}^{+}$decays were also seen for electron transfer from $\mathrm{Mn}$ (II) bound to a four-helix bundle that was not attached to the reaction center [20]. Computer modeling places the four-helix bundle on the periplasmic surface of the reaction center, with the binding site for the porphyrin at a position comparable to the heme-binding site of cytochrome $c_{2}$ [20]. The rate may be limited by the iron-protoporphyrin having an unfavorable orientation when bound to the MPB2 reaction centers resulting in a larger edge-to-edge distance to $\mathrm{P}$ compared to the favorable orientation of bound cytochrome $c_{2}$, or energetic limitations due to a large reorganization energy. Thus the observed slow accumulation of the oxidized iron-protoporphyrin signal in the light may reflect a number of parameters that control electron transfer. Despite the slowness of this process, the spectral changes are observable due to the very slow decay of the iron-protoporphyrin signal, resulting in a continual buildup of the oxidized state during illumination.

Because both proteins are highly pliable, the combination of the four-helix bundle with the reaction center opens the door to an array of novel functions. For example, the fusion of an extramembrane $\alpha$ helix to the $\mathrm{L}$ subunit of the reaction center resulted in the formation of coiled-coils that enabled the directed assembly of reaction centers into multimers [49]. Artificial proteins have been designed with a variety of cofactors participating in specific, self-contained functional features $[3,15,16]$. Similarly, artificially expanding the reaction center template has been accomplished by the attachment of molecules to the surface to enhance light capture and energy transfer [50-54]. Energy transfer has also been demonstrated for the fusion of a fluorescent yellow protein and a light-harvesting protein with the reaction center $[55,56]$, as well as a four-helix bundle with a bound fluorescent cofactor fused to a light-harvesting protein [57]. The fusion of four-helix bundles to reaction centers described here is a unique enhancement of the natural electron transfer functions by allowing redox reactions that are initiated by light. For the MPB2-FeP protein, the redox processes of the porphyrin bound to the four-helix bundle are driven by light excitation of the partner protein rather than the introduction of external chemical oxidants. Such a capability could be useful in hybrid proteins employed in electronic devices [58]. 
In conclusion, our results demonstrate the feasibility of using the fusion of protein domains to create new functions in proteins. The versatility of the four-helix bundle to tightly bind different porphyrins provides a general platform for the design of hybrid complexes capable of a range of lightdriven functions.

Supplementary Information The online version contains supplementary material available at https://doi.org/10.1007/s43630-021-00142-7.

Author contributions The authors have made substantial contributions and approved the contents.

Funding This work was supported by a grant from the National Science Foundation (CHE 1904860) to J.P.A and J.C.W.

Availability of data and material The data are available upon request from the corresponding author.

Code availability Not applicable.

\section{Declarations}

Conflict of interest The authors have no conflicts of interest or competing interests to declare.

\section{References}

1. Nanda, V., \& Koder, R. L. (2010). Designing artificial enzymes by intuition and computation. Nature Chemistry, 2, 15-24.

2. Reetz, M. T. (2013). Biocatalysis in organic chemistry and biotechnology: Past, present, and future. Journal of the American Chemical Society, 135, 12480-12496.

3. Huang, P. S., Boyken, S. E., \& Baker, D. (2016). The coming of age of de novo protein design. Nature, 537, 320-327.

4. Grayson, K. J., \& Anderson, J. L. R. (2018). Designed for life: Biocompatible de novo designed proteins and components. Journal of the Royal Society Interface, 15, 20180472.

5. Sheldon, R. A., \& Woodley, J. M. (2018). Role of biocatalysis in sustainable chemistry. Chemical Reviews, 118, 801-838.

6. Yu, Y., Liu, X., \& Wang, J. (2019). Expansion of redox chemistry in designer metalloenzymes. Accounts of Chemical Research, 52, 557-565.

7. Baker, D. (2019). What has de novo protein design taught us about protein folding and biophysics? Protein Science, 28, 678-683.

8. Korendovych, I. V., \& DeGrado, W. F. (2020). De novo protein design, a retrospective. Quarterly Reviews of Biophysics, 53(e3), $1-33$.

9. Peacock, A. F. A. (2013). Incorporating metals into de novo proteins. Current Opinion in Chemical Biology, 17, 934-939.

10. Yu, F., Cangelosi, V. M., Zastrow, M. L., Tegoni, M., Plegaria, J. S., Tebo, A. G., Mocny, C. S., Ruckthong, L., Qayyum, H., \& Pecoraro, V. L. (2014). Protein design: Toward functional metalloenzymes. Chemical Reviews, 114, 3495-3578.

11. Fry, B. A., Solomon, L. A., Dutton, P. L., \& Moser, C. C. (2016). Design and engineering of a man-made diffusive electron-transport protein. Biochimica et Biophysica Acta Bioenergetics, 1857, 513-521.

12. Hosseinzadeh, P., \& Lu, Y. (2016). Design and fine-tuning redox potentials of metalloproteins involved in electron transfer in bioenergetics. Biochimica et Biophysica Acta Bioenergetics, 1857, 557-581.

13. Farid, T. A., Kodali, G., Solomon, L. A., Lichtenstein, B. R., Sheehan, M. M., Fry, B. A., Bialas, C., Ennist, N. M., Siedlecki, J. A., Zhao, Z., Stetz, M. A., Valentine, K. G., Anderson, J. L. R., Wand, A. J., Discher, B. M., Moser, C. C., \& Dutton, P. L. (2013). Elementary tetrahelical protein design for diverse oxidoreductase functions. Nature Chemical Biology, 9, 826-833.

14. Liu, J., Chakraborty, S., Hosseinzadeh, P., Yu, Y., Tian, S., Petrik, I., Bhagi, A., \& Lu, Y. (2014). Metalloproteins containing cytochrome, iron-sulfur, or copper redox centers. Chemical Reviews, 114, 4366-4469.

15. Mocny, C. S., \& Pecoraro, V. L. (2015). De novo protein design as a methodology for synthetic bioinorganic chemistry. Accounts of Chemical Research, 48, 2388-2396.

16. Lombardi, A., Pirro, F., Maglio, O., Chino, M., \& DeGrado, W. F. (2019). De novo design of four-helix bundle metalloproteins: One scaffold, diverse reactivities. Accounts of Chemical Research, 52, 1148-1159.

17. Mann, S. I., Nayak, A., Gassner, G. T., Therien, M. J., \& DeGrado, W. F. (2021). De novo design, solution characterization, and crystallographic structure of an abiological $\mathrm{Mn}$-porphyrin-binding protein capable of stabilizing a $\mathrm{Mn}(\mathrm{V})$ species. Journal of the American Chemical Society, 143, 252-259.

18. Olson, T. L., Espiritu, E., Edwardraja, S., Simmons, C. R., Williams, J. C., Ghirlanda, G., \& Allen, J. P. (2016). Design of dinuclear manganese cofactors for bacterial reaction centers. Biochimica et Biophysica Acta Bioenergetics, 1857, 539-547.

19. Olson, T. L., Espiritu, E., Edwardraja, S., Canarie, E., Flores, M., Williams, J. C., Ghirlanda, G., \& Allen, J. P. (2017). Biochemical and spectroscopic characterization of dinuclear Mn-sites in artificial four-helix bundle proteins. Biochimica et Biophysica Acta Bioenergetics, 1858, 945-954.

20. Espiritu, E., Olson, T. L., Williams, J. C., \& Allen, J. P. (2017). Binding and energetics of electron transfer between an artificial four-helix Mn-protein and reaction centers from Rhodobacter sphaeroides. Biochemistry, 56, 6460-6469.

21. Allen, J. P., Feher, G., Yeates, T. O., Komiya, H., \& Rees, D. C. (1987). Structure of the reaction center from Rhodobacter sphaeroides R-26: The protein subunits. Proceedings of the National Academy of Sciences of the United States of America, 84, 6162-6166.

22. Hunter, C. N., Daldal, F., Thurnauer, M. C., \& Beatty, J. T. (Eds.) (2009). The purple phototrophic bacteria. Springer-Verlag.

23. Lin, X., Murchison, H. A., Nagaragan, V., Parson, W. W., Allen, J. P., \& Williams, J. C. (1994). Specific alteration of the oxidation potential of the electron donor in reaction centers from Rhodobacter sphaeroides. Proceedings of the National Academy of Sciences of the United States of America, 91, 10265-10269.

24. Calhoun, J. R., Nastri, F., Maglio, O., Pavone, V., Lombardi, A., \& DeGrado, W. F. (2005). Artificial diiron proteins: From structure to function. Biopolymers, 80, 264-278.

25. Maglio, O., Nastri, F., Martin, T., de Rosales, R., Faiella, M., Pavone, V., DeGrado, W. F., \& Lombardi, A. (2007). Diiron-containing metalloproteins: Developing functional models. Comptes Rendus Chimie, 10, 703-720.

26. Chino, M., Maglio, O., Nastri, F., Pavone, V., DeGrado, W. F., \& Lombardi, A. (2015). Artificial diiron enzymes with a de novo designed four-helix bundle structure. European Journal of Inorganic Chemistry, 2015, 3371-3390.

27. Paddock, M. L., Rongey, S. H., Feher, G., \& Okamura, M. Y. (1989). Pathway of proton transfer in bacterial reaction centers: Replacement of glutamic acid 212 in the L subunit by glutamine inhibits quinone (secondary acceptor) turnover. Proceedings of the National Academy of Sciences of the United States of America, 86, 6602-6606. 
28. Goldsmith, J. O., King, B., \& Boxer, S. G. (1996). Mg coordination by amino acid side chains is not required for assembly and function of the special pair in bacterial photosynthetic reaction centers. Biochemistry, 35, 2421-2428.

29. Espiritu, E., Chamberlain, K. D., Williams, J. C., \& Allen, J. P. (2020). Bound manganese oxides capable of reducing the bacteriochlorophyll dimer of modified reaction centers from Rhodobacter sphaeroides. Photosynthesis Research, 143, 129-141.

30. Thielges, M., Uyeda, G., Cámara-Artigas, A., Kálmán, L., Williams, J. C., \& Allen, J. P. (2005). Design of a redox-linked active metal site: Manganese bound to bacterial reaction centers at a site resembling that of photosystem II. Biochemistry, 44, 7389-7394.

31. Korendovych, I. V., \& DeGrado, W. F. (2014). Catalytic efficiency of designed catalytic proteins. Current Opinion in Structural Biology, $27,113-121$

32. Johnson, W. C. (1999). Analyzing protein circular dichroism spectra for accurate secondary structures. Proteins, 35, 307-312.

33. Sreerama, N., \& Woody, R. W. (2000). Estimation of protein secondary structure from circular dichroism spectra: Comparison of CONTIN, SELCON, and CDSSTR methods with an expanded reference set. Analytical Biochemistry, 287, 252-260.

34. Louis-Jeune, C., Andrade-Navarro, M. A., \& Perez-Iratxeta, C. (2012). Prediction of protein secondary structure from circular dichroism using theoretically derived spectra. Proteins, 80, 374-381.

35. Micsonai, A., Wien, F., Kernya, L., Lee, Y. H., Goto, Y., Réfrégiers, M., \& Kardos, J. (2015). Accurate secondary structure prediction and fold recognition for circular dichroism spectroscopy. Proceedings of the National Academy of Sciences of the United States of America, 112, E3095-E3103.

36. Chen, X., Zaro, J., \& Shen, W. C. (2013). Fusion protein linkers: Property, design, and functionality. Advanced Drug Delivery Reviews, 65, 1357-1369.

37. Calhoun, J. R., Kono, H., Lahr, S., Wang, W., DeGrado, W. F., \& Saven, J. G. (2003). Computational design and characterization of a monomeric helical dinuclear metalloprotein. Journal of Molecular Biology, 334, 1101-1115.

38. Calhoun, J. R., Liu, W., Spiegel, K., Dal Peraro, M., Klein, M. L., Valentine, K. G., Wand, A. J., \& DeGrado, W. F. (2008). Solution NMR structure of a designed metalloprotein and complementary molecular dynamics refinement. Structure, 16, 210-215.

39. Williams, J. C., \& Allen, J. P. (2009). Directed modification of reaction centers from purple bacteria. In C. N. Hunter, F. Daldal, M. C. Thurnauer, \& J. T. Beatty (Eds.), The purple phototrophic bacteria (pp. 337-353). Springer-Verlag.

40. Park, K., Perczel, A., \& Fasman, G. D. (1992). Differentiation between transmembrane helices and peripheral helices by the deconvolution of circular dichroism spectra of membrane proteins. Protein Science, 1, 1032-1049.

41. Sreerama, N., \& Woody, R. W. (2004). On the analysis of membrane protein circular dichroism spectra. Protein Science, 13, 100-112.

42. Cordova, J. M., Noack, P. L., Hilcove, S. A., Lear, J. D., \& Ghirlanda, G. (2007). Design of a functional membrane protein by engineering a heme-binding site in Glycophorin A. Journal of the American Chemical Society, 129, 512-518.

43. Jones, M. R. (2009). Structural plasticity of reaction centers from purple bacteria. In C. N. Hunter, F. Daldal, M. C. Thurnauer, \& J. T. Beatty (Eds.), The purple phototrophic bacteria (pp. 295-321). Springer-Verlag.

44. Bylina, E. J., \& Youvan, D. C. (1988). Directed mutations affecting spectroscopic and electron transfer properties of the primary donor in the photosynthetic reaction center. Proceedings of the National Academy of Sciences of the United States of America, 85, 7226-7230.

45. McDowell, L. M., Gaul, D., Kirmaier, C., Holten, D., \& Schenck, C. C. (1991). Investigation into the source of electron transfer asymmetry in bacterial reaction centers. Biochemistry, 30, $8315-8322$.

46. Camara-Artigas, A., Magee, C., Goetsch, A., \& Allen, J. P. (2002). The structure of the heterodimer reaction center from Rhodobacter sphaeroides at $2.55 \AA$ A resolution. Photosynthesis Research, 74, 87-93.

47. Axelrod, H. L., Abresch, E. C., Okamura, M. Y., Yeh, A. P., Rees, D. C., \& Feher, G. (2002). X-ray structure determination of the cytochrome $c_{2}$ : Reaction center electron transfer complex from Rhodobacter sphaeroides. Journal of Molecular Biology, 319, 501-515.

48. Kálmán, L., \& Maróti, P. (1997). Conformation-activated protonation in reaction centers of the photosynthetic bacterium Rhodobacter sphaeroides. Biochemistry, 36, 15269-15276.

49. Swainsbury, D. J. K., Harniman, R. L., Di Bartolo, N. D., Liu, J., Harper, W. F. M., Corrie, A. S., \& Jones, M. R. (2016). Directed assembly of defined oligomeric photosynthetic reaction centres through adaptation with programmable extra-membrane coiledcoil interfaces. Biochimica et Biophysica Acta Bioenergetics, 1857, 1829-1839.

50. Nabiev, I., Rakovich, A., Sukhanova, A., Lukashev, E., Zagidullin, V., Pachenko, V., Rakovich, Y. P., Donegan, J. F., Rubin, A. B., \& Govorov, A. O. (2010). Fluorescent quantum dots as artificial antennas for enhanced light harvesting and energy transfer to photosynthetic reaction centers. Angewandte Chemie International Edition, 49, 7217-7221.

51. Milano, F., Tangorra, R. R., Hassan Omar, O., Ragni, R., Operamolla, A., Agostiano, A., Farinola, G. M., \& Trotta, M. (2012). Enhancing the light harvesting capability of a photosynthetic reaction center by a tailored molecular fluorophore. Angewandte Chemie International Edition, 51, 11019-11023.

52. Dutta, P. K., Levenberg, S., Loskutov, A., Jun, D., Saer, R., Beatty, J. T., Lin, S., Liu, Y., Woodbury, N. W., \& Yan, H. (2014). A DNAdirected light-harvesting/reaction center system. Journal of the American Chemical Society, 136, 16618-16625.

53. Dutta, P. K., Lin, S., Loskutov, A., Levenberg, S., Jun, D., Saer, R., Beatty, J. T., Liu, Y., Yan, H., \& Woodbury, N. W. (2014). Reengineering the optical absorption cross-section of photosynthetic reaction centers. Journal of the American Chemical Society, 136, $4599-4604$.

54. Hassan Omar, O., la Gatta, S., Tangorra, R. R., Milano, F., Ragni, R., Operamolla, A., Argazzi, R., Chiorboli, C., Agostiano, A., Trotta, M., \& Farinola, G. M. (2016). Synthetic antenna functioning as light harvester in the whole visible region for enhanced hybrid photosynthetic reaction centers. Bioconjugate Chemistry, 27, 1614-1623.

55. Grayson, K. J., Faries, K. M., Huang, X., Qian, P., Dilbeck, P., Martin, E. C., Hitchcock, A., Vasilev, C., Yuen, J. M., Niedzwiedzki, D. M., Leggett, G. J., Holten, D., Kirmaier, C., \& Hunter, C. N. (2017). Augmenting light coverage for photosynthesis through YFPenhanced charge separation at the Rhodobacter sphaeroides reaction centre. Nature Communications, 8, 13972.

56. Liu, J., Friebe, V. M., Frese, R. N., \& Jones, M. R. (2020). Polychromatic solar energy conversion in pigment-protein chimeras that unite the two kingdoms of (bacterio)chlorophyll-based photosynthesis. Nature Communications, 11, 1542.

57. Mancini, J. A., Kodali, G., Jiang, J., Reddy, K. R., Lindsey, J. S., Bryant, D. A., Dutton, P. L., \& Moser, C. C. (2017). Multi-step excitation energy transfer engineered in genetic fusions of natural and synthetic light-harvesting proteins. Journal of the Royal Society Interface, 14, 20160896.

58. Milano, F., Punzi, A., Ragni, R., Trotta, M., \& Farinola, G. M. (2019). Photonics and optoelectronics with bacteria: Making materials from photosynthetic microorganisms. Advanced Functional Materials, 29, 1805521. 